\title{
SPECTRAL ENERGY DISTRIBUTIONS OF PG QUASARS
}

\author{
G. Neugebauer, D. B. Sanders, B. T. Soifer, S. Phinney \\ Division of Physics, Mathematics and Astronomy \\ California Institute of Technology \\ Downs Lab of Physics, 320-47 \\ Pasadena, CA 91125
}

\author{
R. F. Green \\ National Optical Astronomy Observatories \\ P. O. Box 26732
}

Tucson, $A Z$ 85726-6732

\begin{abstract}
Between $10^{13}-10^{17} \mathrm{~Hz}$ the continua of all PG quasars can be described in the most general terms by a model consisting of two broad peaks of thermal radiation. There is no evidence for energetically significant nonthermal radiation in this frequency range in the continua of the PG quasars.

We have compiled continuum observations for PG quasars from $6 \mathrm{~cm}$ to $2 \mathrm{~K}_{\mathrm{e}} \mathrm{V}$, including IRAS data for all these objects and new ground-based infrared data at $10 \mu \mathrm{m}$ for many of these quasars. Sixty-three of the PG quasars were detected by IRAS in at least one band. The overall energy distributions for these sixty-three PG quasars are shown in Figure 1.

Observationally, the bumps are the well-known "big blue bump" at $\sim 10^{15}-10^{17} \mathrm{~Hz}(0.003-0.3 \mu \mathrm{m})$ and an infrared bump at $\sim 10^{12.5}-10^{14.3} \mathrm{~Hz}-(1.5-100 \mu \mathrm{m})$ with a shallow minimum between the two bumps occurring within a small range at $\sim 10^{14.5} \mathrm{~Hz}(1 \mu \mathrm{m})$. There is a weak correlation between the luminosities in the two bumps, but for objects with $\mathrm{L}_{\text {bol }}>10^{12} \mathrm{~L}_{\odot}$, the relative strength of the blue bump is the same in most of the quasars, while the total luminosity contained in the infrared bump varies from 0 - $50 \%$ of that in the blue. In $\sim 50 \%$ of PG quasars the infrared bump luminosity is $\gtrsim 20 \%$ of that in the blue bump. For $P G$ quasars less luminous than $10^{12} \mathrm{~L}_{\odot}$, both the infrared and blue bump components seem to disappear.
\end{abstract}

There is no obvious connection between the strengths of the bumps and whether or not the quasar is radio quiet or radio loud. For both radio quiet quasars $\left(\nu f_{\nu}(\log \nu=14.5) / \nu f_{\nu}(\log \nu=10) \gtrsim 10^{5.5}\right)$ that represent $\sim 90 \%$ of the total number of PG objects, and steep-spectrum radio loud quasars $\left(\nu \mathrm{f}_{\nu}(\log \nu=\right.$ $\left.14.5) / \nu f_{\nu}(\log \nu=10) \lesssim 10^{2.5}\right)$ there is a fairly abrupt downturn in the continuum distribution at wavelengths longward of $100 \mu \mathrm{m}$ which most likely intersects with the radio continuum just longward of $1 \mathrm{~mm}$. Only in the compact, flat-spectrum radio loud sources $(5 / 109)$ does the radio appear to extrapolate smoothly to the $100 \mu \mathrm{m}$ emission.

A simple explanation of the gross energy distribution is that the bumps represent thermal emission from either dust or gas in the quasar. The blue, high frequency bump results from gas within tens of AUs of the central quasar engine, presumably in the accretion disk, which is heated to temperatures in the neighborhood of $\sim 10^{5} \mathrm{~K}$. The infrared bump consists of contributions from a distribution of dust at distances from one 


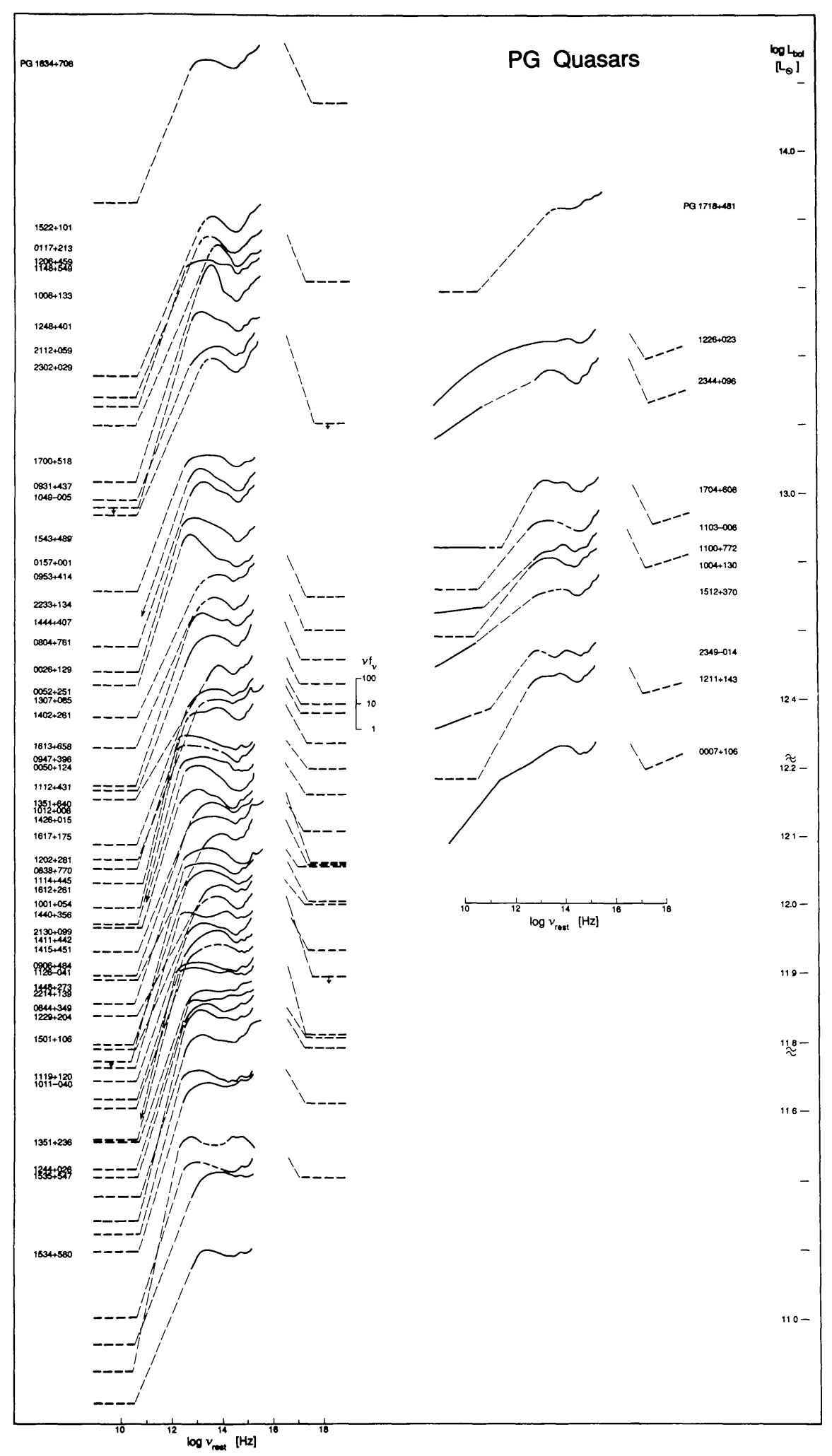


parsec to a few kilo-parsecs from the central source; the dust has a range of temperatures from $\sim 50$ to $\sim 1000 \mathrm{~K}$. The mass of dust required for the infrared bump can be estimated with reasonable assumptions about the grain properties to vary from $\sim 0.01 \mathrm{M}_{\odot}$ at $2 \mu \mathrm{m}$ to about $10^{5} \mathrm{M}_{\odot}$ at $60 \mu \mathrm{m}$. The small range in the cross-over frequency presumably reflects the fact that the temperature of the dust represented near $1 \mu \mathrm{m}$ exceeds the sublimation temperature of the dust grains, and may be taken as further confirmation of the hypothesis of thermal emission. The fairly small range in the relative sizes of the bumps may be taken as an indication that the covering factors in most of the PG quasars is similar.

The observations are consistent with any nonthermal continuum connected to the radio emission being independent of the thermal emission. In radio quiet quasars the bolometric luminoisty of such a continuum represents only a small fraction $(<10 \%)$ of the total bolometric luminosity, while in radio loud quasars it represents a more conspicuous, at some frequencies even dominant, fraction of the luminosity. A caveat that should be added to this picture is that the fraction of the infrared continuum at wavelengths greater than a few microns which varies on a time scale less that $\sim 1$ year must be associated with a nonthermal continuum.

Figure 1. Energy distributions plotted as $\log \nu \mathrm{f}_{\nu}$ vs. $\log \nu$, for sixty-three PG quasars from $\sim 10^{10} \mathrm{~Hz}$ to $\sim 10^{18} \mathrm{~Hz}$. The vertical scale corresponds to bolometric luminosity for the quasar in units of solar luminosity for assumed Hubble constant $\mathrm{H}_{\mathrm{O}}=75 \mathrm{~km} \mathrm{~s}^{-1} \mathrm{Mpc}^{-1}$. Each quasar is plotted so that the value of $\nu \mathrm{f}_{\nu}$ at $\log \nu=14.5$ corresponds to the bolometric luminosity. The name of the quasar is printed at that same vertical level. Thick solid lines represent a smooth fit to the observed data points. In the radio, where most objects have been observed only at $6 \mathrm{~cm}$, a thick horizontal dashed line represents an assumed $\mathrm{f}_{\nu} \propto \nu^{-1}$ spectrum for both radio quiet and steep-spectrum radio loud quasars. At $\mathrm{X}$-ray wavelengths, the thick dashed line slope through the $2 \mathrm{Kev}$ data point assumes spectral slopes of -1 and 0 for radio quiet and radio loud objects respectively. Thin dashed lines connect the observed far-infrared data to the extrapolated radio data. The shape of the 'big blue bump' between $\log \nu_{\text {rest }} \simeq 15.5-16.5 \mathrm{~Hz}$ is unknown; a thin dashed line extrapolates the high frequency edge of the bump at $\log \nu=16.5 \mathrm{~Hz}$ (with an assumed height equal to the last observed UV data point near $\log \nu=15.5 \mathrm{~Hz}$ ) to the X-ray data.

\section{DISCUSSION}

B. WILLS Do you really require a power law in radio quiet quasars? If you do not include it, what difference does this make to the estimates of dust mass?

SOIFER We do not require an underlying power law. If such a power law exists, it is substantially less than the observed continuum in the infrared. Our dust mass estimates are based on the assumption that the entire IR continuum is due to dust emission, so that any underlying non-thermal continuum would reduce the inferred masses of dust.

WARD I notice that in several of the energy distributions you showed the $\mathrm{X}$-ray flux (in $v F_{v}$ ) is substantially below the level of the infrared/optical/ultraviolet continuum. This is in contrast with the situation observed in, for example, the Piccinotti sample of AGN.

Are the $X$-ray data soft energy observations? If so, is this evidence for high intrinsic column densities possibly correlated with the strength of the 2-4 $\mu$ infrared bumps?

SOIFER The $X$-ray fluxes were taken from Einstein data at $2 \mathrm{keV}$. The spectral shape in the $X$-ray is assumed to be the canonical shape inferred by Wilkes, Elvis, etc. 